\title{
CONFIABILIDADE DE UM PROTÓTIPO PARA AVALIAÇÃO DAS MEDIDAS DO DESEMPENHO
}

\author{
RELIABILITY OF A PROTOTYPE FOR EVALUATION OF PERFORMANCE MEASURES \\ FIABILIDAD DE UN PROTOTIPO PARA LA EVALUACIÓN DE LAS MEDIDAS DE RENDIMIENTO
}

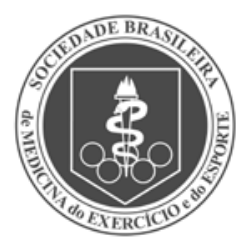

Artigo Original

Original ARTICLE

Artículo Original
Marcos André Rodrigues da Silva Júnior (Educação Física)

Helivelton Gomes Barbosa² (Ciências da Computação)

João Constantino da Silva Neto 3 (Ciências da Computação)

Paulo Moreira Silva Dantas ${ }^{4}$ (Educação Física)

1. Instituto Federal de Alagoas, Palmeira dos Índios, AL, Brasil. 2. Universidade Federal de Alagoas UFAL, Maceió, AL, Brasil. 3. Universidade Federal de Alagoas UFAL, Arapiraca, AL, Brasil. 4. Universidade Federal do Rio Grande do Norte - UFRN, Natal, RN, Brasil.

\section{Correspondência:}

Instituto Federal de Alagoas Campus Palmeira dos Índios (Setor de Educação Física). Av. Alagoas, s/n - Palmeira de Fora. Palmeira dos Índios, AL, Brasil. marcosandrejunior@gmail.com

\section{RESUMO}

Introdução: Mensurar o desempenho humano é um desafio, principalmente devido ao fator multidimensional do movimento. Os instrumentos, em sua maioria, avaliam apenas uma dimensão do referido desempenho. Objetivo: Verificar a concordância dos resultados das medidas do desempenho, velocidade média, velocidade final, aceleração média, força, trabalho e potência média, calculadas pelo protótipo e compará-las com os resultados calculados por um pacote estatístico considerado o padrão ouro. Métodos: A amostra constituiu-se de atletas paralímpicos de basquete $(n=09)$ e atletas de caratê $(n=31)$ e todos assinaram o termo de consentimento. Foi desenvolvido e utilizado um protótipo para avaliar as medidas do desempenho, e os resultados calculados pelo protótipo foram comparados com os resultados calculados pelo pacote estatístico utilizando as mesmas fórmulas. Resultados: Todas as variáveis calculadas pelo protótipo não apresentaram diferenças significativas quando comparadas com os resultados calculados pelo pacote estatístico. Conclusão: O dispositivo pode ser usado para mensurar as medidas do desempenho, com retorno imediato dos resultados e sem necessidade de um computador, desde que observados os critérios descritos.

Descritores: destreza motora; trabalho; potência.

\section{ABSTRACT}

Introduction: Measuring human performance is a challenge, mainly due to the multidimensional movement factor. Most instruments only evaluate one dimension of said performance. Objective: To verify the concordance of the results of performance measures, average speed, final speed, average acceleration, force, work and average power, calculated by the prototype and compare them with the results calculated by a statistical package considered as gold standard. Methods: The sample consisted of Paralympic basketball athletes $(n=09)$ and karate athletes $(n=31)$ and all participants signed a consent form. A prototype was developed and used to evaluate performance measures, and the results calculated by the prototype were compared with the results calculated by the statistical package using the same formulas. Results: All variables calculated by the prototype showed no significant differences when compared with the results calculated by the statistical package. Conclusion: The device can be used to evaluate performance measures with immediate return of results and without need of a computer, provided that described criteria are satisfied.

Keywords: motor skills; work; potency.

\section{RESUMEN}

Introducción: Medir el rendimiento humano es un desafío, principalmente en virtud del factor multidimensional del movimiento. En su mayoría, los instrumentos solo evalúan una dimensión de dicho rendimiento. Objetivo: Verificar la concordancia de los resultados de las medidas de rendimiento, velocidad mediana, velocidad final, aceleración mediana, fuerza, trabajo y potencia mediana, calculadas por el prototipo y compararlas con los resultados calculados por un paquete estadístico considerado el patrón oro. Métodos: La muestra fue constituida por atletas paralímpicos de baloncesto $(n=09)$ y atletas de karate $(n=31)$, y todos firmaron la declaración de consentimiento. Fue desarrollado y utilizado un prototipo para evaluar las medidas de rendimiento, y los resultados calculados por el prototipo fueron comparados con los resultados calculados por el paquete estadístico utilizando las mismas fórmulas. Resultados: Todas las variables calculadas por el prototipo no presentaron diferencias significativas cuando comparadas con los resultados calculados por el paquete estadístico. Conclusión: El dispositivo puede ser usado para valorar las medidas de rendimiento, con retorno inmediato de los resultados y sin necesidad de una computadora, siempre que observados los criterios descritos.

Descriptores: destreza motora; trabajo; potencia. 


\section{INTRODUÇÃO}

Avaliar aspectos relacionados à motricidade humana é algo inerente às ciências do movimento humano. Por isso, utilizar métodos que sejam menos invasivos, de fácil aplicação, com menor custo, que apresentem resultados em tempo real, e valores quantitativos tão bons quanto os métodos considerados de "padrão ouro", é essencial para a área das ciências do movimento e sua consequente evolução'.

Diversos instrumentos têm sido utilizados para a avaliação das medidas do desempenho, e a maior parte desses consegue mensurar uma ou duas dessas variáveis do desempenho. Alguns destes instrumentos necessitam de aparelhagem e mão-de-obra especializada para sua utilização adequada, tornando oneroso o processo de obtenção dos resultados das avaliações e ainda, muitas vezes, não são apresentados em tempo real ${ }^{2,3}$.

Assim sendo, protótipos que sejam capazes de avaliar diversas medidas relacionadas ao desempenho simultâneo, que sejam de fácil uso e apresentem resultados imediatos, certamente contribuirão para o aprimoramento da performance humana. Deste modo, conseguir desenvolver um protótipo capaz de avaliar o indivíduo ambientado, possuindo ou não limitações, em sua atividade é um desafio para as ciências do movimento humano, esse se torna maior quando os instrumentos de avaliação buscam a portabilidade, facilidade de uso, o baixo custo e alta precisão.

Além da dificuldade do desenvolvimento, a confiabilidade dos resultados mensurados é fundamental e deve apresentar uma relação de concordância, não apenas uma associação com os resultados considerados "padrão ouro" que são aspectos bem diferentes ${ }^{4,5}$. Portanto, o presente estudo tem como objetivo verificar a concordância dos resultados das medidas do desempenho Velocidade Média, Velocidade Final, Aceleração Média, Força, Trabalho e Potência Média calculadas pelo protótipo desenvolvido e compará-las com os resultados calculados por um pacote de análise estatística.

\section{MÉTODOS}

A amostra constitui-se de 40 atletas voluntários do gênero masculino, sendo 31 deles de caratê e nove de basquete em cadeira de rodas. O estudo faz parte do projeto que está cadastrado no Comitê de Ética da Universidade Federal do Rio Grande do Norte, RN, Brasil, sob o número 576/11. Todos os voluntários deram seu consentimento para participação no estudo no Termo de Consentimento Livre e Esclarecido.

Foi utilizada uma balança digital com precisão de $50 \mathrm{~g}$ para aferir a Massa Total (MT) e todos os indivíduos foram pesados com o material que utilizam para sua prática esportiva. Para os atletas de basquete foi medida a massa da cadeira e do indivíduo vestido com sua roupa de prática separadamente, depois foi somado o valor da massa corporal com o da cadeira de rodas, foram pesados sentados sobre a plataforma da balança. Para os atletas de caratê; a massa foi medida com o quimono, posição ereta, no centro da balança e braços ao longo do tronco.

Para aferir as medidas do desempenho empregou-se um Protótipo (Proto) desenvolvido pelo autor do presente estudo, com placa de circuito eletrônico, micro controlador, sensor de inclinação em três eixos (SM), laser (Ls), fotoemissor (FE), resistor dependente de luz (LDR), botões de pressão (BT) e um visor de LCD (LCD) no qual os resultados das medidas do desempenho são visualizados após a avaliação.

Para medida das variáveis seguiu-se o seguinte procedimento:

1. O protótipo era ligado verificando-se o funcionamento (testando as cinco opções descritas no decorrer do texto);

2. Uma marcação no solo era realizada (uma linha de partida de onde o atleta ou segmento corporal deveria estar posicionado);
3. O LDR e o Ls eram posicionados a um metro da linha de partida, um de frente para o outro de forma que o Ls ilumine o orifício da caixa do LDR;

4. O FE era posicionado à frente da linha de partida, a cinco metros para evitar colisão;

5. O SM era posicionado no centro da região occipital nos paratletas de Basquete e ligeiramente acima do maléolo lateral do membro inferior dominante nos atletas de caratê (figura 1).

6. A Massa Total foi aferida Massa Total (MT) em kg e seu valor foi inserido no Protótipo.

Os atletas foram informados como seria realizado o teste e somente foram adaptados ao Protótipo após não haver mais questionamentos. O teste foi realizado e os dados anotados em uma planilha.

O Proto mensura as seguintes medidas do desempenho: Tempo de Reação (TReac); Tempo de Movimento (TMov); Tempo de Resposta (TResp). As três medidas são mensuradas usando a função contida na programação do Proto chamada "millis", que retorna ao sistema o tempo em milissegundos desde o momento que o Proto foi ligado. Assim, se pode calcular o TeReac subtraindo o intervalo de tempo entre o acendimento do FE e a captação do início do movimento pelo SM do tempo que o Proto foi ligado. O TResp calcula-se subtraindo o intervalo de tempo entre o acendimento do FE e a passagem do avaliado pelo LDR. O TMov é a diferença entre TResp e TReac. A aferição do tempo medido pelo Proto foi calibrado utilizando instrumento para estudo da mecânica dos sólidos: estática, cinemática e dinâmica; MRU e MRUA (aceleração positiva e aceleração negativa); inércia; conservação da energia; impulsão; quantidade de movimento; colisões.

As medidas do desempenho Velocidade Média (VelMed), Velocidade Final (VelFinal), Aceleração Média (AcelMed), Força (F), Trabalho (Trab) e Potência Média (PotMed) são calculadas a partir das fórmulas descritas a seguir e são medidas seguindo o Sistema Internacional de Unidade (SI), essas fórmulas estão na programação do Proto.

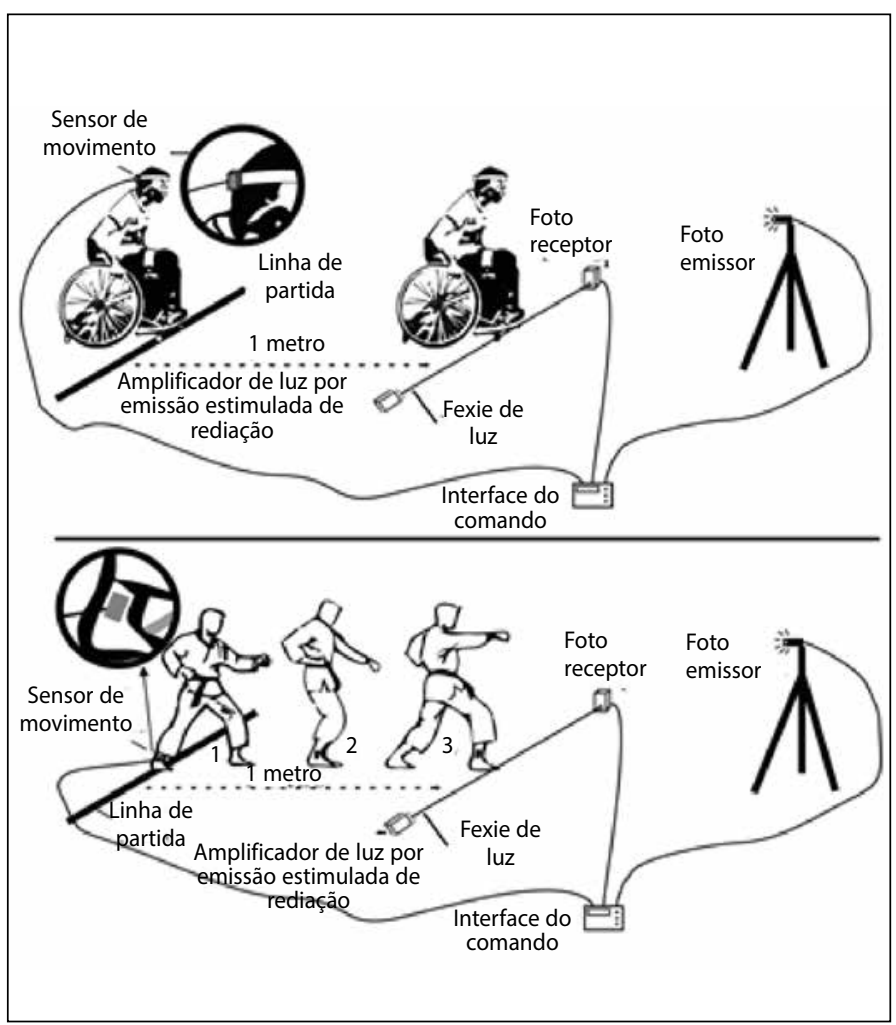

Figura 1. Ilustração do Protótipo e procedimentos de avaliação para aquisição das medidas do desempenho em atletas de basquete e caratê. 
As fórmulas abaixo também serão utilizadas pelo PE para cálculo das medidas do desempenho e os resultados obtidos pelos dois, Proto e PE, serão comparados.

$$
\begin{aligned}
\text { VelMed } & =\frac{\Delta s}{\Delta t} \\
\text { VelFinal } & =\text { Vo }+a \times t \\
\text { AcelMed }=>S & =S O+\text { Vot }+\frac{a t^{2}}{2} \\
\text { Força } & =m \times a \\
\text { Trab } & =F \times d \\
\text { PotMed } & =\frac{\text { Trab }}{t}
\end{aligned}
$$

Onde: $\Delta s=$ Distância percorrida em (m); $\Delta t=$ Tempo do deslocamento em (s); Vo = Velocidade Inicial em (m/s); a = Aceleração em $\left(\mathrm{m} / \mathrm{s}^{2}\right) ; \mathrm{S}=$ Deslocamento percorrido em $(\mathrm{m}) ; \mathrm{So}=$ Deslocamento inicial em (m); $m$ = massa deslocada em (kg); F = Força em (N); Trab = Trabalho em (J); PotMed = Potência Média em (w).

A normalidade e igualdade das variâncias dos dados foram verificadas utilizando-se os testes de Shapiro-Wilk e Leneve. Utilizando esses critérios identificou-se como paramétricas apenas as variáveis VelMed, VelFinal e AcelMed para o grupo basquete $(n=09)$ e VelMed para caratê $(n=31)$ e de caratê e basquete $(n=40)^{6}$.

Para comparar os dados calculados pelo Proto e pelo PE para as variáveis paramétricas utilizou-se o teste $t$ de pareado e para as não paramétricas o Teste de Mann-Whitney ${ }^{6}$. Como medida de confiabilidade numérica entre o Proto e PE foi usado o método de análise de concordância entre métodos de Bland-Altman ${ }^{4,5}$. O intervalo de confiança adotado foi de 95\%. O Pacote Estatístico (PE) utilizado foi o SPSS 20.0 e SigmaPlot 12. Para as variáveis que tiveram suas diferenças em zero não foi gerado o gráfico representativo, por não haver, pela evidência do resultado, a necessidade do teste de hipótese.

\section{RESULTADOS}

Na tabela 1 estão descritos os dados com valores de média de desvio padrão (DP) e significância ( $p$ ) das diferenças entre os valores calculados

\begin{tabular}{|c|c|c|c|c|}
\hline Diferença das Variáveis & $\mathbf{N}$ & Med & DP & $p$ \\
\hline Diferença entre VelMed (Proto e PE) & 9 & 0,00 & 0,00 & $0,975^{\dagger}$ \\
\hline Diferença entre VelFinal (Proto e PE) & 9 & 0,00 & 0,00 & $0,751^{\dagger}$ \\
\hline Diferença entre AcelMed (Proto e PE) & 9 & $-0,01$ & 0,05 & $0,916^{\dagger}$ \\
\hline Diferença entre F (Proto e PE) & 9 & 2,08 & 6,73 & 0,972 \\
\hline Diferença entre Trab (Proto e PE) & 9 & 2,08 & 6,73 & 0,972 \\
\hline Diferença entre PotMed (Proto e PE) & 9 & $-3,22$ & 10,54 & 0,972 \\
\hline Diferença entre VelMed (Proto e PE) & 31 & $-0,01$ & 0,07 & $0,895^{\dagger}$ \\
\hline Diferença entre VelFinal (Proto e PE) & 31 & 0,94 & 5,39 & 0,965 \\
\hline Diferença entre AcelMed (Proto e PE) & 31 & $-0,18$ & 1,85 & 0,965 \\
\hline Diferença entre $\mathrm{F}$ (Proto e PE) & 31 & $-31,51$ & 126,87 & 0,965 \\
\hline Diferença entre Trab (Proto e PE) & 31 & $-31,51$ & 126,87 & 0,965 \\
\hline Diferença entre PotMed (Proto e PE) & 31 & $-264,42$ & 1292,39 & 0,965 \\
\hline Diferença entre VelMed (Proto e PE) & 40 & $-0,00$ & 0,06 & $0,986^{\dagger}$ \\
\hline Diferença entre VelFinal (Proto e PE) & 40 & 0,73 & 4,74 & 0,817 \\
\hline Diferença entre AcelMed (Proto e PE) & 40 & $-0,15$ & 1,62 & 0,946 \\
\hline Diferença entre $\mathrm{F}$ (Proto e PE) & 40 & $-24,89$ & 112,01 & 0,985 \\
\hline Diferença entre Trab (Proto e PE) & 40 & $-24,89$ & 112,01 & 0,985 \\
\hline Diferença entre PotMed (Proto e PE) & 40 & $-205,65$ & 1138,88 & 0,977 \\
\hline
\end{tabular}
pelo Proto e pelo PE. Os dados são apresentados por categoria sendo: os atletas de basquete $(n=09)$, atletas de caratê $(N=31)$, e atletas de caratê e

Tabela 1. Descrição dos valores de Média (Med), Desvio Padrão (DP), significância (p) das diferenças entre Proto e PE para os três grupos (basquete $n=09$, caratê $n=31$ e caratê e basquete $n=40$ ).

$\leq 0,05$. Teste $t$ pareado, demais: Mann-Whitney. basquete juntos $(n=40)$. Sequencialmente, a análise de concordância foi demonstrada através de gráficos de dispersão utilizando o método de Bland-Atman para as variáveis F, Trab e PotMed que apresentaram diferenças entre Proto e PE maiores que zero. Esse método permite avaliar a extensão das diferenças entre os métodos (Proto e PE), além da dispersão em relação à média, bem como tendências e outliers ${ }^{4,5}$.

Os dados da tabela 1 apresentam valores que, independente do grupo avaliado, os resultados obtidos quando os dados são calculados pelo Proto e pelo PE são comparados, não apresentam diferenças significativas entre si.

Quando observadas as variáveis VelMed, VelFinal e AcelMed os valores da diferença média foram iguais a zero, mostrando que não houve uma diferença entre os resultados calculados pelo protótipo e os resultados calculados pelo PE, esse fato ocorreu quando analisamos os dados independente da categoria analisada (basquete $n=09$, caratê $\mathrm{n}=31$ e caratê e basquete $\mathrm{n}=40$ ).

Os dados das variáveis F, Trab e PotMed apresentaram uma diferença entre Proto e PE acima de zero, contudo, essas diferenças não são estatisticamente significativas conforme demonstram os valores de $p$ estas variáveis independentemente do grupo avaliado.

A seguir nas figuras 2, 3 e 4, são apresentados os gráficos de dispersão utilizando o método de Bland-Altman para as variáveis $F$, Trab e PotMed essa abordagem permite, de forma visual, verificar a concordância das médias dos dois grupos, possibilitando assim, avaliar as discordâncias com as medidas avaliadas ${ }^{4,5}$.
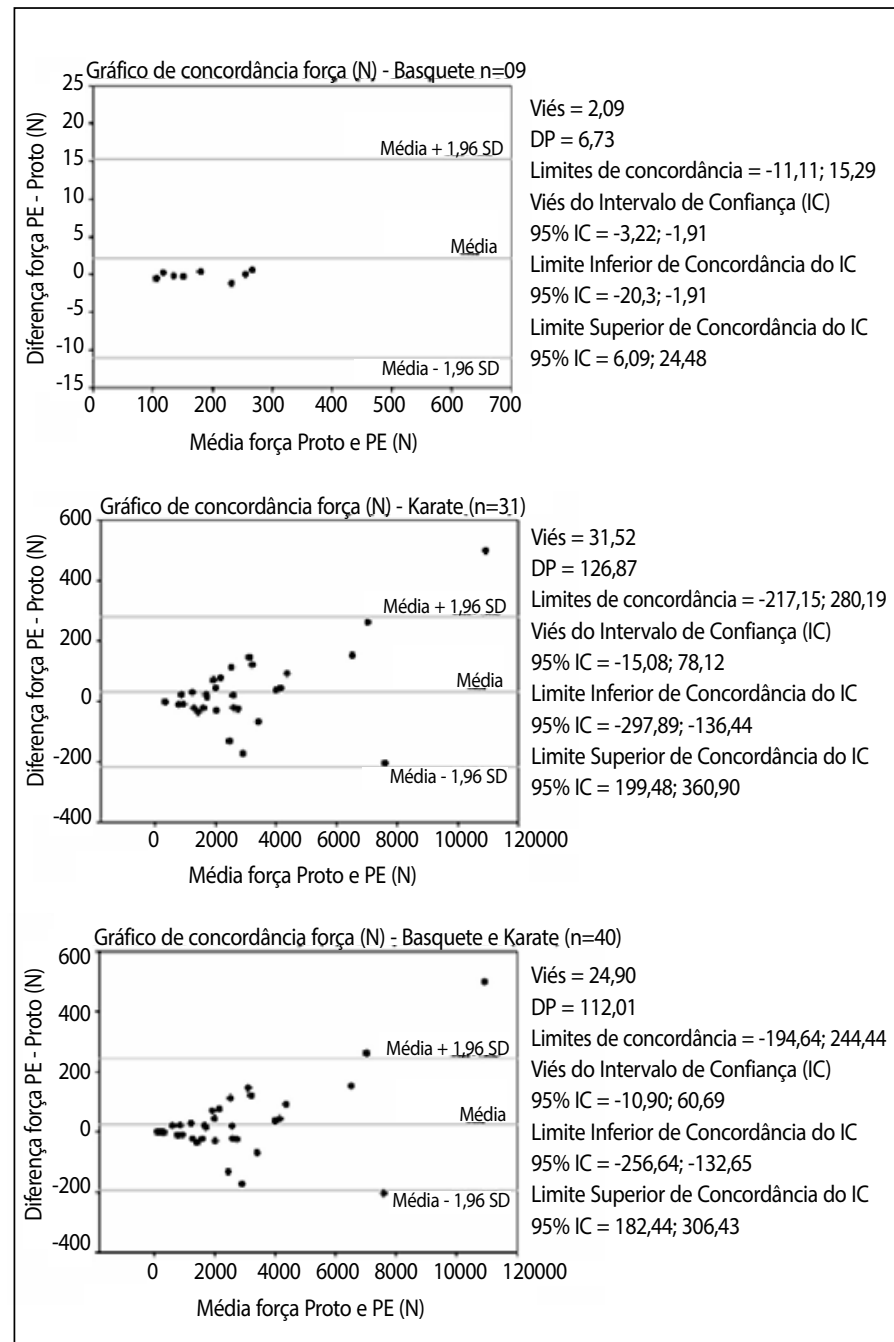

Figura 2. Concordância da variável Força (F) calculada pelo Protótipo e pelo PE para basquete $(n=09)$, caratê $(n=31)$ e caratê e basquete $(n=40)$, respectivamente. 


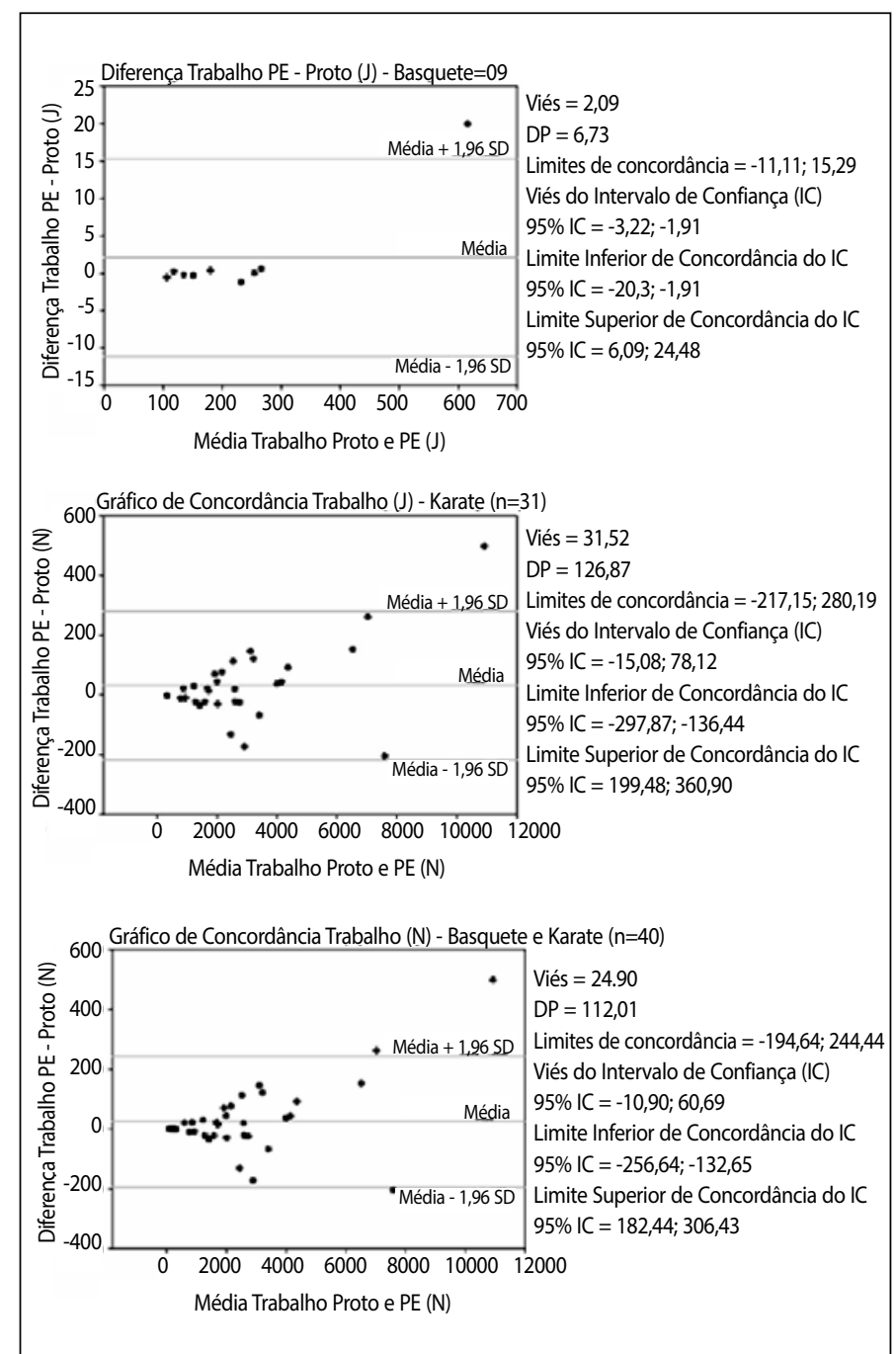

Figura 3. Concordância da variável Trabalho (Trab) calculada pelo Protótipo e pelo PE para basquete $(n=09)$, caratê $(n=31)$ e caratê e basquete $(n=40)$, respectivamente.

\section{DISCUSSÃO}

Com a finalidade de verificar se o Protótipo é capaz de calcular as variáveis VelMed, VelFinal, F, Trab e PotMed foi necessário realizar uma comparação dos resultados utilizando um "padrão ouro", neste caso, utilizou-se um Pacote Estatístico largamente utilizado em pesquisas científicas, pois quando se trata de movimento humano, vários métodos de avaliação têm sido utilizados para análise de informações que auxiliem a compreensão do gesto corporal e de seus segmentos. No entanto, até o momento não existe uma forma que solucione o problema da avaliação do ser humano, particularmente durante a realização de uma determinada atividade. Tais dificuldades de aferição provêm do fator multidimensional do movimento humano relacionado à ação muscular, movimento articular e ao controle e qualidade do movimento. A maioria dos instrumentos e métodos utilizados para avaliação do movimento humano, mensura apenas um aspecto destes, impossibilitando uma compreensão universal do movimento ${ }^{1,7}$.

Dentre os métodos mais utilizados encontram-se a avaliação cinematográfica de vídeos e fotos. Apesar de serem métodos precisos, pois possibilitam um detalhamento do movimento, sua utilização é demorada, requer instalação de marcadores, alto investimento com softwares e hardwares, além de treinamento para processamento e análise dos dados computacionais ${ }^{1,7-10}$.

Buscando contribuir para o desenvolvimento da análise do movimento humano, foi desenvolvido um Protótipo que em sua programação

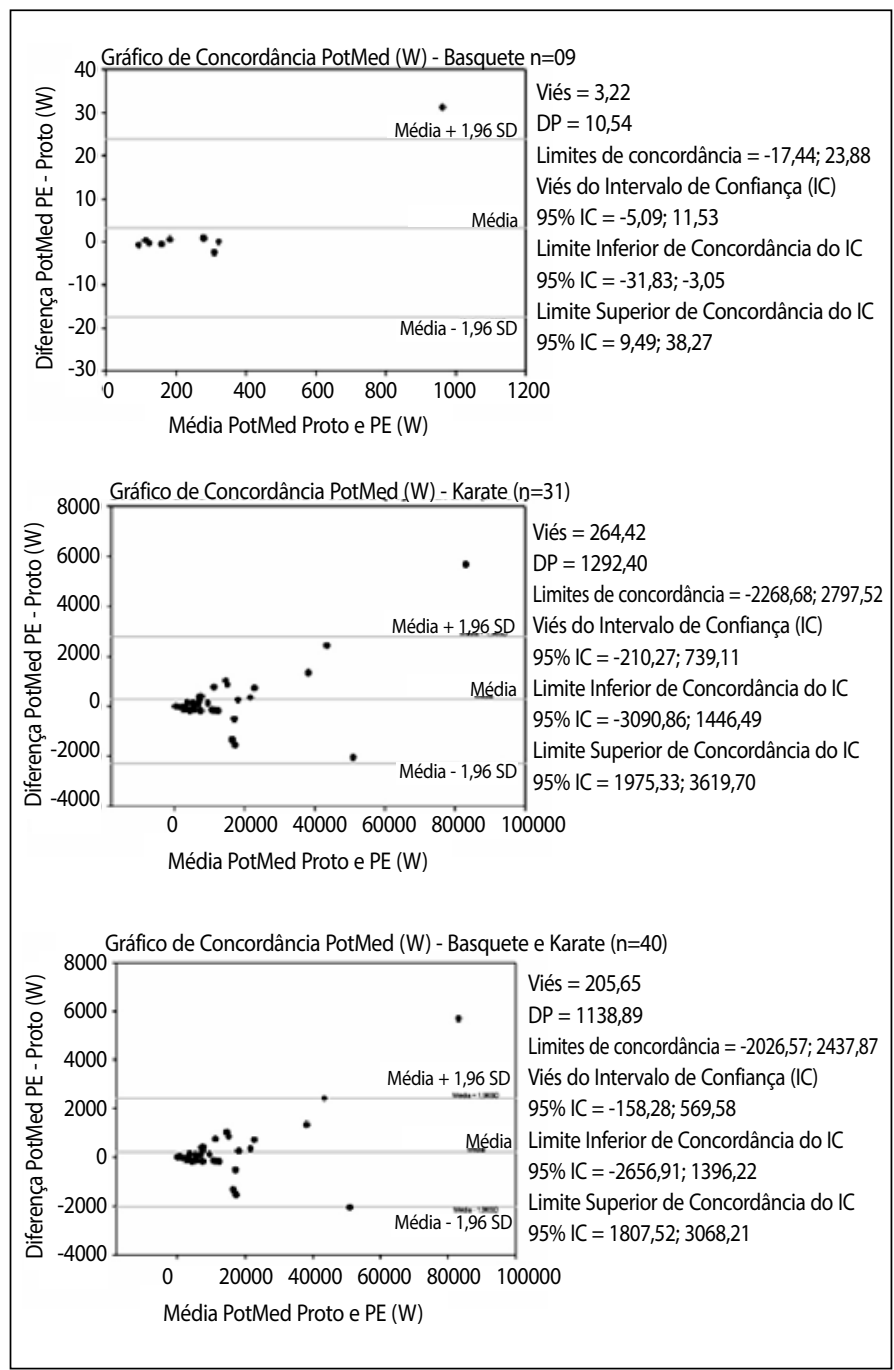

Figura 4. Concordância da variável Potência Média (PotMed) calculada pelo Protótipo e pelo PE para basquete $(n=09)$, caratê $(n=31)$ e caratê e basquete $(n=40)$, respectivamente.

possui as equações necessárias para realizar os cálculos das medidas do desempenho Velocidade Média e Final, Aceleração Média, Força, Trabalho e Potência Média em um único teste, mas a fim de verificar se os resultados obtidos, de fato, são fidedignos, essas equações por sua vez, foram utilizadas no Pacote Estatístico, considerado o "Padrão Ouro" e os resultados foram comparados ao Protótipo a fim de verificar sua fidedignidade.

Vários estudos relacionados às medidas do desempenho apresentam uso de instrumentação para a mensuração das variáveis analisadas, a exemplo encontram-se estudos com atletas de caratê ${ }^{7}$, atletas de kung $\mathrm{fu}^{9}$, surfistas $^{10}$, jogadores de futsal ${ }^{11}$, atletas de basquete, ginástica artística, não atletas ${ }^{12}$ e jogadores de voleibol ${ }^{13}$. Nestes estudos foi constatado que o uso de instrumentos cinematográficos, aparelhos com interface acoplada a computadores convencionais, equipamentos de alto custo que necessitam de treinamento específico para sua aplicação e que consomem tempo pós-avaliação para tratamento dos resultados obtidos os estudos, além de resultados que podem sofrer influência devido à forma de utilização dos instrumentos de avaliação ${ }^{10}$. Apesar de serem instrumentos científicos reconhecidos e utilizados na comunidade científica e com resultados válidos, eles nem sempre medem as variáveis do desempenho durante a atividade física do avaliado, indo de encontro ao princípio da especificidade do movimento, não expressando assim, resultados relacionados à sua prática esportiva ou atividades do dia a dia.

Quando a avaliação possui aplicações terapêuticas, como no caso de pessoas paraplégicas, o foco passa a ser a independência funcional, 
retorno aos hábitos de vida, reintegração do indivíduo em seu ambiente social, além de melhorias nas funções musculares objetivando a troca de posturas e o manuseio da cadeira de rodas ${ }^{14}$, testes que utilizam métodos válidos, entretanto consomem tempo pré-avaliação, se baseiam em estimativas de cargas e não possuem semelhança na execução dos movimentos do avaliado ${ }^{15,16}$.

Em todos os estudos citados, tecnologias que possuem alto desempenho na obtenção de dados frequentemente possuem limitações relacionadas à mobilidade do atleta, provenientes de uma série de dispositivos e fios, fazendo com que os testes não utilizem gestos motores condizentes com sua prática desportiva. Outros métodos, por sua vez, precisam de tempo para edição de imagem ou dados, não os disponibilizando rapidamente para que possa ocorrer uma intervenção na preparação técnica dos atletas.

Neste estudo, o Protótipo permite uma maior amplitude de movimento possibilitando que o avaliado realize a maioria dos movimentos inerentes as suas atividades. Os dados são processados imediatamente após a realização do teste e os resultados são expostos no LCD da interface de comando seguidamente após a avaliação, disponibilizando resultados imediatos ao avaliado e ao treinador diferentemente das outras formas de obtenção de resultados apresentados, que utilizam processamentos pós teste, fato que aumenta o hiato entre a obtenção do resultado e o processamento, fazendo com que o profissional da área perca tempo precioso.

Quando os resultados obtidos pelo Protótipo foram analisados e estes foram comparados com os resultados do Pacote Estatístico, verificou-se não haver diferenças significativas em nenhuma das variáveis analisadas, além do que, as variáveis VelMed, VelFinal e AcelMed calculadas pelo Protótipo apresentaram uma diferença igual a zero quando comparadas com os valores calculados pelo Pacote Estatístico. Mesmo as variáveis Força, Trabalho e Potência Média apresentando uma diferença superior a zero, quando comparadas com o Protótipo, estes resultados não foram estatisticamente significativos, independentemente se os dados foram analisados para o grupo de basquete, caratê ou os dois grupos juntos.

O gráfico de dispersão de Bland-Altman retorna uma compreensão visual dos valores, no qual sua maioria encontra-se próximo ao valor médio de cada variável para todos os grupos. Independentemente dos atletas avaliados participarem ou não de uma modalidade esportiva paralímpica, os resultados apresentaram-se satisfatórios para ambos os grupos, mostrando que, seguindo os critérios para análise das medidas do desempenho descritas, o Protótipo pode ser utilizado para medir as variáveis VelMed, VelFInal, Acelmed, F, Trab e PotMed de forma fidedigna.

O Protótipo desenvolvido e validado neste estudo pode ser uma alternativa viável, pois não é necessário um computador para a obtenção dos resultados, uma vez que a própria interface do protótipo já apresenta os resultados imediatamente após o teste ser realizado. Além do que, o protótipo pode funcionar com baterias de nove volts o que lhe dará a possibilidade de não depender de uma fonte de energia para ligar o aparelho, podendo aplicar os testes no local onde o atleta está ambientado.

Além da fidedignidade e dos resultados serem apresentados imediatamente após a avaliação, o protótipo pode ser utilizado para verificar medidas do desempenho importantes como força, trabalho e potência de membros superiores em pessoas com paraplegia. A avaliação dessas variáveis é importante para a prescrição de exercícios para aquela população, uma vez que, estudos indicam que o treinamento regular de potência parece incrementar as habilidades motoras de membros superiores, tendo relação direta com a amenização ou até superação de sequelas relacionadas a lesão medular ${ }^{14,17}$. Ainda, os níveis de força e resistência tem relação direta com a prevenção de úlceras de decúbito e a capacidade de impulsionar a cadeira de rodas e consequente independência do portador de paraplegia. Logo, a avaliação dessas variáveis do desempenho humano favorecerão a prescrição de atividades físicas mais precisas para que o indivíduo possa retornar as suas atividades diárias e ter mais independência14,17.

\section{CONCLUSÃO}

Com base na amostra, o estudo permitiu concluir que o Protótipo desenvolvido consegue calcular de forma precisa as medidas do desempenho Velocidade Média, Velocidade Final, Aceleração Média, Força, Trabalho e Potência Média, podendo ser utilizado em grupos de pessoas diferentes sem perder eficiência na mensuração, desde que observados os procedimentos descritos. Novos estudos podem ser realizados em outras populações. O protótipo pode ser uma ferramenta para mensuração das variáveis do desempenho humano podendo ser utilizado por profissionais da área das ciências da saúde que necessitem da avaliação dessas variáveis para melhoria da prescrição de exercício físico e apresentação imediata dos resultados para suas práticas profissionais.

Todos os autores declararam não haver qualquer potencial conflito de interesses referente a este artigo.

CONTRIBUIÇÕES DOS AUTORES: Cada autor contribuiu individual e significativamente para o desenvolvimento do manuscrito. MARSJ (0000-0002-7296-7419)* e PMSD (0000-0002-5684-5884)* foram os principais contribuintes na redação e análise estatística do manuscrito. HGB (0000-0002-0179-4979)* e JCSN (0000-00032685-9190)* atuaram na revisão bibliográfica e desenvolvimento do hardware e software do Protótipo.

\section{REFERÊNCIAS}

1. Trew M, Everett T. Movimento Humano. $4^{a}$ ed. São Paulo: Premier; 2010.

2. Borysiuk Z. Psychomotor reactions in fencing dependence of stimuli type. Rev Bras Cineantropom Desempenho Hum. 2008;10:223-9.

3. Vaguetti $\mathrm{CAO}$, Roesler $\mathrm{H}$, Andrade A. Tempo de reação simples auditivo e visual em surfistas com diferentes níveis de habilidade: comparação entre atletas profissionais, amadoras e praticantes. Rev Bras Med Esporte. 2007;13(2):81-5.

4. Hirakata VN, Camey SA. Análise de concordância entre métodos de Bland-Altman. Rev HCPA. 2009;29(3):261-8

5. Bland JM, Altman DG. Measuring agreement in method comparison studies. Stat Methods Med Res. 1999;8(2):135-60

6. Field A. Descobrindo a Estatística usando SPSS. $2^{a}$ ed. Porto Alegre: Artmed; 2009.

7. Mori S, Ohtani Y, Imanaka K. Reaction times and anticipatory skills of karate athletes. Hum Mov Sci. 2002;21(2):213-30.

8. Amadio AC, Costa PHL, Sacco ICN, Serrão JC, Araujo RC, Mochizuki L, et al. Introdução à biomecânica para análise do movimento humano: descrição e aplicação dos métodos de medição. Rev Bras Fisioter. 1999;3(2):41-54.

9. Pinto Neto O Magini M, Saba MMF. Análise Cinemática de um Movimento de Kung-Fu: a importância de uma apropriada interpretação física para dados obtidos através de câmeras rápidas. Rev Bras Ensino Fís. 2006;28(2):235-9.
10. Vaghetti $\mathrm{CAO}$, Roesler $\mathrm{H}$, Andrade $\mathrm{A}$. Tempo de reação simples auditivo e visual em surfistas com diferentes níveis de habilidade: comparação entre atletas profissionais, amadores e praticantes. Rev Bras Med Esporte. 2007;13(2):81-5.

11. Chagas MH, Leite CMF, Ugrinowitsch H, Benda RN, Menzel HJ, Souza PRC et al. Associação entre tempo de reação e de movimento em jogadores de futsal. Ver Bras Educ Fís Esp. 2005;19(4):269-75.

12. Bruzi AT, Fialho JVAP, Fonseca FS, Ugrinowitsch H. Comparação do tempo de reação entre atletas de basquetebol, ginástica artística e não atletas. Rev Bras Ciênc Esporte. 2013;35(2):469-80.

13. Barcelos JL, Morales AP, Maciel RN, dos Anjos MMa, da Silva VF. Tempo de prática: estudo comparativo do tempo de reação motriz entre jogadoras de voleibol. Fit Perf J. 2009;8(2):103-9.

14. Sartori J, Neuwald MF, Bastos VH, Silva JG, Mello MP, Freitas MRG, et al. Reabilitação física na lesão traumática da medula espinhal: relato de caso. Rev Neuroc. 2009;17:364-79.

15. Bortolloti LF, Tsukamoto HF. Efeitos do treinamento físico sobre a força muscular em paraplégicos. Rev Neurocienc. 2011;19(3):462-71

16. Dias RMR, Cyrino ES, Salvador EP, Nakamura FY, Pina FLC, Oliveira AR. Impacto de oito semanas de treinamento com pesos sobre a força muscular de homens e mulheres. Rev Bras Med Esporte. 2005;11(4):224-8.

17. Gorgatti MG, Costa RFd. Atividade Física Adaptada: qualidade de vida para pessoas com necessidades especiais. $2^{\text {a }}$ ed. Barueri, SP: Manole; 2008. 